\title{
Monitoreo del arrecife coralino Meager Shoal, Parque Nacional Cahuita, Costa Rica (sitio CARICOMP)
}

\author{
Ana C. Fonseca E. ${ }^{1}$, Eva Salas ${ }^{1,2}$ \& Jorge Cortés ${ }^{1,2}$ \\ 1 Centro de Investigación en Ciencias del Mar y Limnología (CIMAR), Universidad de Costa Rica, 2060 San José, \\ Costa Rica; afonseca@cariari.ucr.ac.cr \\ 2 Escuela de Biología, Universidad de Costa Rica, 2060 San José, Costa Rica. Fax (506) 207-3280
}

Recibido 31-X-2005. Corregido 16-III-2006. Aceptado 23-V-2006.

\begin{abstract}
Monitoring of the Meager Shoal coral reef, Cahuita National Park, Costa Rica (CARICOMP site). The coral reefs at Cahuita National Park, Caribbean coast of Costa Rica, specifically at the CARICOMP site Meager Shoal, have been monitored since 1999. Complete data sets from 2000 and 2004 have shown that live coral cover has increased less than $3 \%$ (from 15 to $17 \%$ ), but non-coralline algae cover has increased much (63 to $74 \%$ ) and coralline algae cover has decreased (17 to $5 \%$ ) significantly. The proportion of affected colonies by diseases, injuries and bleaching decreased from $24 \%$ in 2000 to $10 \%$ in 2004 , but the difference was not statistically significant. Densities of the urchin Diadema antillarum increased, and are probably help to maintain the macroalgae biomass low, while those of Echinometra viridis decreased significantly. The coral reef at Cahuita National Park continues to be impacted by chronic terrigenous sediments and does not show a significant recovery since the late 1970's. Rev. Biol. Trop. 54 (3): 755-763. Epub 2006 Sept. 29.
\end{abstract}

Key words: coral reef, monitoring, CARICOMP, Cahuita, Caribbean, Costa Rica.

Los arrecifes coralinos de todo el mundo están siendo amenazados por una combinación de impactos naturales y antropogénicos (Richmond 1993, Hughes et al. 2003, Pandolfi et al. 2003). Los impactos naturales son intensos pero espaciados, y estos niveles de disturbio intermedio pueden contribuir a mantener la biodiversidad (Connell 1978). Por el contrario los disturbios humanos no siempre son tan intensos pero son crónicos y no le dan oportunidad a los ecosistemas de recuperarse y mantener su función (Richmond 1993, Nyström et al. 2000, Nyström y Folke 2001, Hughes et al. 2005).

A raíz de esta situación, se están estableciendo programas de monitoreo para evaluar esos impactos, y distinguir entre problemas reales y variaciones temporales naturales (Richmond 1993), e identificar el grado de resiliencia de los ecosistemas marino costeros (Nyström et al. 2000, Nyström y Folke 2001,
Hughes et al. 2003, 2005). En el Caribe el programa científico internacional colaborativo llamado "Productividad Marino-Costera del Caribe (CARICOMP)" ha estado monitoreando manglares, pastos marinos y arrecifes coralinos desde 1990 (CARICOMP 1997). Este programa consiste en realizar mediciones estándar y sinópticas de ecosistemas costeros en los sitios en mejor estado de conservación de cada país, a una profundidad de $10 \mathrm{~m}$, para detectar cambios en los mismos y distinguir entre disturbios naturales y humanos. En Costa Rica el arrecife Meager Shoal en el Parque Nacional Cahuita se ha estado monitoreando siguiendo ese protocolo. Esta área protegida se creó en 1970 y tiene un territorio marino de 22400 ha, de las cuales 600 ha están cubiertas por el arrecife coralino de franja más desarrollado y más estudiado de Costa Rica (Cortés 1998, Cortés y Jiménez 2003).

Los arrecifes coralinos de Cahuita han sido ampliamente estudiados desde los años 
70 usando diferentes metodologías (Cortés y Jiménez 2003, Fonseca 2003). El objetivo de este estudio es presentar los resultados del monitoreo en la estación permanente de CARICOMP, Meager Shoal, compararlos con otros estudios de la región y dar algunas recomendaciones para su manejo.

\section{MATERIALES Y MÉTODOS}

Descripción del sitio: Los arrecifes coralinos del Parque Nacional Cahuita están localizados en la sección sur de la costa Caribe de Costa Rica (Fig. 1). El arrecife consiste de tres crestas, la cresta principal es de $5 \mathrm{~km}$ de largo y corre paralela al frente de Punta Cahuita, y las otras dos en ambos lados de la punta. Un sistema angosto de canales y espolones alcanza profundidades de hasta $15 \mathrm{~m}$ en el frente arrecifal de la cresta principal. Varios arrecifes de parche pequeños ocurren en la laguna y hay varios bancos carbonatados mar afuera del lado sur de Punta Cahuita (Risk et al. 1980, Cortés y Risk 1985). Este arrecife también presenta la mayor biodiversidad, con tres especies de hidrocorales (Cortés 1992), treinta y un especies de corales escleractíneos (Cortés y Guzmán 1985), seis especies de corales azooxantelados (Cortés, en prep.) y diecinueve especies de octocorales (Guzmán y Cortés 1985). Las corrientes fluyen hacia el sureste con algunos remolinos en dirección opuesta. Estas corrientes transportan sedimentos terrestres derivados de tierras deforestadas corriente arriba, a lo largo de ríos y costas (Cortés y Risk 1985, Cortés 1994). Estos sedimentos son la causa principal de la degradación del arrecife coralino a lo largo de los últimos 50 años, combinado con el aumento de otros disturbios naturales y antropogénicos (Fonseca y Cortés 1998, Cortés y Jiménez 2003, Fonseca 2003).

El arrecife coralino del Parque Nacional Cahuita es considerado un activo económico con un valor de por lo menos $\$ 1.4$ millones al año (N. Blair et al. datos sin publicar). La población de Cahuita aumentó de 3000 (Bermúdez 1992) a 4000 habitantes en los últimos 13 años, y recibe más de 50000 turistas al año (Fonseca y Cortés 1998, MINAE 2005, com. pers.). Las actividades económicas principales en la comunidad son el turismo y la pesca.

Los pescadores de Cahuita tienen conocimiento de tres bancos carbonatados mar afuera: Sea Lal (943'16.0" N, 82 46'33.0" W), Little Shoal $\left(9^{\circ} 43^{\prime} 50.6^{\prime \prime} \mathrm{N}, 82^{\circ} 47^{\prime} 51.5^{\prime \prime} \mathrm{W}\right)$ y Meager Shoal (943'50" N, 8248'32" W). El banco carbonatado de Meager Shoal está localizado a 1 $\mathrm{km}$ frente a la playa de Puerto Vargas, al sur de la cresta principal de Cahuita (Fig. 1). Tiene un área circular de $10000 \mathrm{~m}^{2}$, la base del banco está a $10 \mathrm{~m}$ y el tope a $7 \mathrm{~m}$ de profundidad. Los corales se concentran en el tope del banco y las especies dominantes son Agaricia agaricites, Porites astreoides, Siderastrea siderea y Montastraea faveolata. Otras especies de coral encontradas son Mycetophyllia spp., Madracis decactis, Dichocoenia stokesii y Siderastrea radians. Este banco arrecifal está rodeado de barro terrestre que es fácilmente resuspendido por las corrientes prevalecientes y fuerte oleaje, aún así es uno de los arrecifes en mejor estado a esa profundidad.

Métodos: Con el fin de comparar los resultados del monitoreo en Costa Rica con los de otros países del Caribe, se siguió la

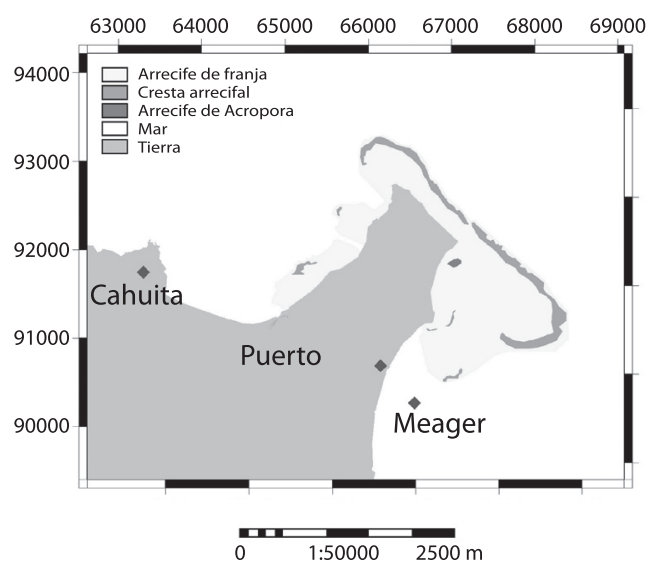

Fig. 1. Sitio de estudio, Parque Nacional Cahuita, Limón, Costa Rica.

Fig. 1. Study site, Parque Nacional Cahuita, Limón, Costa Rica. 
metodología de Productividad Marino Costera del Caribe (CARICOMP 1997, 2002). Este protocolo se estableció en la región desde 1990. En Costa Rica se hicieron intentos de establecer una estación permanente en 1997, pero sin la colaboración de la comunidad se perdieron las varillas que marcaban el sitio (Cortés 1998). Fue hasta 1999 que se marcó una localidad diferente y desde entonces se ha estado evaluando una vez al año, sin embargo se tienen datos completos sólo para el 2000 y el 2004. Al mismo tiempo, la comunidad local fue educada sobre la importancia de los arrecifes coralinos y entrenada para colaborar con los científicos para recolectar parte de la información, especialmente las medidas físicas semanales.

El banco arrecifal de Meager Shoal fue seleccionado para establecer dos estaciones con cinco transectos permanentes de $10 \mathrm{~m}$ en cada una. La cobertura relativa del arrecife se evaluó con el método de la cadena y la densidad de erizos con el método de cinturón de $1 \mathrm{~m}$ de ancho. Además se realizaron cuatro transectos (20 x $2 \mathrm{~m}$ ) para determinar la densidad de colonias coralinas y la incidencia de enfermedades. En el 2000, se tomaron 10 muestras de algas para estimar la biomasa de éstas. En el 2000 y el 2003 se recolectaron dos muestras de agua con un volumen de 21 cada una para dar un estimado de la materia particulada en suspensión (MPS). La composición y densidad de peces fue evaluada en el 2004 por medio de cinco transectos de cinturón de $30 \times 2 \mathrm{~m}$, metodología AGRRA, y con el método del buzo ambulante para completar el inventario (un buceo de 60 min de 9:30 a 10:30 am), metodología REEF. La temperatura del agua se ha estado registrando permanentemente desde 1999 con un sensor submarino marca Stow Away programado para tomar medidas cada 15 min, y sólo en el 2000 se estuvo registrando cada tres meses la salinidad con un refractómetro y la turbidez con un disco Secchi (CARICOMP 1997, 2002). Los datos sobre cobertura relativa del arrecife y la proporción de enfermedades fue normalizada y analizada utilizando ANOVA de una vía (Underwood 1997).
La estación permanente para la medida de temperatura atmosférica y precipitación se encuentra en la casa de guardaparques de

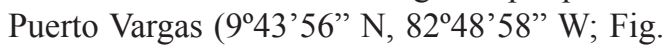
1). La temperatura atmosférica se evaluó de 1996 a 1999 con un termómetro de mercurio (Max-Min mercury), y del 2000 al presente con un sensor Stow Away que toma medidas cada $15 \mathrm{~min}$.

\section{RESULTADOS}

Temperatura atmosférica y precipitación: La temperatura atmosférica osciló en los últimos 10 años entre 22.1 y $30.9{ }^{\circ} \mathrm{C}$. Los meses más calientes fueron de mayo a junio y de setiembre a octubre. La precipitación mensual promedio fue de 215 a $307 \mathrm{~mm}$ (Cuadro 1). En el año 2003 se registró el rango más amplio de temperatura, con las temperaturas mínimas más bajas y las temperaturas máximas más altas, y la menor precipitación promedio. En el 2001 se registró la precipitación más alta.

Temperatura, salinidad y turbidez del agua arrecifal: La temperatura del agua varió entre 23.59 y $31{ }^{\circ} \mathrm{C}$. Los meses más calientes fueron mayo, junio y octubre y la

CUADRO 1

Temperatura atmosférica y precipitación en Puerto Vargas, Limón, Costa Rica (1996-2004)

TABLE 1

Atmospheric temperature and precipitation levels, Puerto Vargas, Limón, Costa Rica (1996-2004)

$\begin{array}{cccc}\text { Año } & \begin{array}{c}\text { Temperatura } \\ \text { mínima }\left({ }^{\circ} \mathrm{C}\right)\end{array} & \begin{array}{c}\text { Temperatura } \\ \text { máxima }\left({ }^{\circ} \mathrm{C}\right)\end{array} & \begin{array}{c}\text { Precipitación } \\ \text { promedio }(\mathrm{mm})\end{array} \\ 1996 & 23.5 & 30.2 & 279.9 \\ 1999 & 22.9 & 29.4 & 234.4 \\ 2000 & 22.4 & 29.0 & 252.7 \\ 2001 & 22.7 & 29.7 & 307.1 \\ 2002 & - & - & - \\ 2003 & 22.1 & 30.9 & 214.7 \\ 2004 & - & - & 301.7\end{array}$


temperatura máxima se obtuvo en el 2003 (Cuadro 2). La salinidad fue de $36.9 \pm 0.87$ psu en el $2000(n=5)$ y la profundidad de Secchi de $3.5 \pm 2.2 \mathrm{~m}(\mathrm{n}=8)$.

Cobertura relativa del sustrato arrecifal: La cobertura de coral se mantuvo baja, con un aumento menor al $3 \%$ del 2000 al 2004, sin embargo las algas no coralinas aumentaron $23 \%(\mathrm{~F}=6.9, \mathrm{gl}=1, \mathrm{p}<.05)$ y las algas coralinas disminuyeron $11 \%(\mathrm{~F}=$ $17.9, \mathrm{gl}=1, \mathrm{p}<.05)$. La cobertura total de algas resultó mayor que la cobertura de coral vivo y aumentó entre el 2000 y el 2004 (ANOVA, $\mathrm{p}<.05$ ) (Cuadro 3).

Enfermedades de coral: La densidad media de colonias de coral en el 2000 fue de 4 colonias $/ \mathrm{m}^{2}$, y en el 2004 disminuyó a 3 colonias $/ \mathrm{m}^{2}$. Las especies de coral dominantes fueron $A$. agaricites, $P$. astreoides y $S$. siderea. La proporción de colonias afectadas por enfermedades, daños y blanqueamiento disminuyó de $24 \%$ en 2000 a $10 \%$ en 2004 , pero la diferencia no fue significativa (ANOVA, $\mathrm{p}>$.05) (Cuadro 4).

En ambos años dominaron las enfermedades de Plaga Blanca (WPD) y la de Manchas Negras (DSD-1). Las enfermedades afectaron principalmente colonias de S. siderea, y el

\section{CUADRO 2}

Temperatura del agua $\left({ }^{\circ} \mathrm{C}\right)$ en Meager Shoal, Cahuita, Limón, Costa Rica (1999-2004)

\section{TABLE 2}

Water temperature $\left({ }^{\circ} \mathrm{C}\right)$ in Meager Shoal, Cahuita, Limón, Costa Rica (1999-2004)

$\begin{array}{lccc} & \text { Promedio } & \text { Mínimo } & \text { Máximo } \\ 1999 & 27.77 & 25.88 & 29.62 \\ 2000 & 27.28 & 25.51 & 30.96 \\ 2001 & 24.60 & 23.59 & 27.11 \\ 2002 & - & - & - \\ 2003 & 29.12 & 28.26 & 31.28 \\ 2004 & 28.18 & 26.65 & 30.04\end{array}$

CUADRO 3

Cobertura relativa del sustrato arrecifal en Meager Shoal

TABLE 3

Relative covering of reef substrate in Meager Shoal

$\begin{array}{lcc} & 2000(\mathrm{n}=10) & 2004(\mathrm{n}=10) \\ \text { Coral vivo } & 14.59 \pm 6.56 & 16.89 \pm 9.46 \\ \text { Algas coralinas } & 16.84 \pm 4.16 & 5.02 \pm 5.17 \\ \text { Algas no coralinas } & 46.09 \pm 12.11 & 68.83 \pm 16.84 \\ \text { Total de algas } & 62.93 \pm 10.54 & 73.85 \pm 14.72 \\ \text { Coralimorfarios } & 0.26 \pm 0.83 & 0.00 \\ \text { Zoantidos } & 0.03 \pm 0.07 & 0.00 \\ \text { Mileporinos } & 0.00 & 0.00 \\ \text { Gorgónidos } & 0.00 & 0.31 \pm 0.99 \\ \text { Esponjas } & 11.26 \pm 3.62 & 3.88 \pm 3.38 \\ \text { Otros } & 10.92 \pm 4.93 & 5.06 \pm 4.24\end{array}$

CUADRO 4

Condición general de las colonias coralinas (prom $\pm D S ; n=4)$

TABLE 4

General condition of coral colonies (mean $\pm S D ; n=4)$

$\begin{array}{lcc} & 2000 & 2004 \\ \text { \% Saludables } & 76.50 \pm 19.76 & 89.01 \pm 9.19 \\ \text { \% Enfermas } & 2.51 \pm 1.90 & 4.20 \pm 3.41 \\ \text { \% Blanqueadas } & 14.14 \pm 11.56 & 4.93 \pm 3.68 \\ \text { \% Dañadas } & 6.85 \pm 6.78 & 1.87 \pm 3.73 \\ \begin{array}{l}\text { \% Enfermedad de } \\ \text { plaga blanca }\end{array} & 0.24 \pm 0.28 & 3.34 \pm 3.36 \\ \begin{array}{l}\text { \% Enfermedad de } \\ \text { manchas negras-I }\end{array} & 2.27 \pm 1.94 & 0.86 \pm 1.43\end{array}$

blanqueamiento se encontró principalmente en $S$. siderea y $P$. astreoides. No se encontraron enfermedades por Banda Negra (BBD), Banda Amarilla (YBD), Manchas Negras (DSD-II), Banda Blanca (WBD) ni Aspergillosis (ASP) (Cuadros 5 y 6 ).

Erizos: La densidad de Diadema antillarum y Eucidaris tribuloides resultaron bajas en Meager Shoal, sin embargo hubo un pequeño aumento de la primer especie. La densidad de Echinometra 
CUADRO 5

Condición de las colonias coralinas por especie en el $2000(n=4)$.

TABLE 5

Condition of coral colonies by species, year $2000(n=4)$

\% Saludables \% Enfermas \% Blanqueadas \% Dañadas \% Plaga blanca \% Manchas

\begin{tabular}{|c|c|c|c|c|c|c|}
\hline Diploria clivosa & 100.00 & 0.00 & 0.00 & 0.00 & 0.00 & 0.00 \\
\hline Montastraea cavernosa & 100.00 & 0.00 & 0.00 & 0.00 & 0.00 & 0.00 \\
\hline Siderastrea siderea & 61.54 & 6.67 & 18.46 & 13.33 & 1.02 & 5.64 \\
\hline Porites astreoides & 83.33 & 0.00 & 15.33 & 1.33 & 0.00 & 0.00 \\
\hline Agaricia agaricites & 98.88 & 0.00 & 1.12 & 0.00 & 0.00 & 0.00 \\
\hline Stephanocoenia intersepta & 100.00 & 0.00 & 0.00 & 0.00 & 0.00 & 0.00 \\
\hline Siderastrea radians & 100.00 & 0.00 & 0.00 & 0.00 & 0.00 & 0.00 \\
\hline Porites porites & 100.00 & 0.00 & 0.00 & 0.00 & 0.00 & 0.00 \\
\hline Leptoseris cucullata & 93.33 & 6.67 & 0.00 & 0.00 & 6.67 & 0.00 \\
\hline Colpophyllia natans & 50.00 & 0.00 & 50.00 & 0.00 & 0.00 & 0.00 \\
\hline
\end{tabular}

CUADRO 6

Condición de las colonias coralinas por especie en el 2004 (n=4)

TABLE 6

Condition of coral colonies by species, year $2004(n=4)$

$\%$ Saludables \% Enfermas \% Blanqueadas \% Dañadas \% Plaga blanca $\begin{gathered}\% \text { Manchas } \\ \text { negras-I }\end{gathered}$

$\begin{array}{lcccccc}\text { Diploria clivosa } & 100.00 & 0.00 & 0.00 & 0.00 & 0 & 0 \\ \text { Montastraea cavernosa } & 93.33 & 0.00 & 0.00 & 6.67 & 0 & 0 \\ \text { Siderastrea siderea } & 82.61 & 6.96 & 6.96 & 3.48 & 4.35 & 2.61 \\ \text { Porites astreoides } & 95.21 & 0.60 & 4.19 & 0.00 & 0.60 & 0 \\ \text { Agaricia humilis } & 100.00 & 0.00 & 0.00 & 0.00 & 0 & 0 \\ \text { Agaricia agaricites } & 97.87 & 1.60 & 0.53 & 0.00 & 1.60 & 0 \\ \text { Madracis decactis } & 100.00 & 0.00 & 0.00 & 0.00 & 0 & 0 \\ \text { Dichocoenia stockesii } & 100.00 & 0.00 & 0.00 & 0.00 & 0 & 0\end{array}$

viridis, disminuyó significativamente del 2000 al 2004 (ANOVA, $\mathrm{p}<.05$ ) (Cuadro 7).

Peces de arrecife: La densidad de peces en el 2004 fue baja, $2.0 \mathrm{ind} / 100 \mathrm{~m}^{2}$, y sólo se encontró una especie de pez cirujano (Acanthurus coeruleus) y una especie de mero (Cephalopholis cruentata), con un tamaño promedio de 15 y $12 \mathrm{~cm}$ respectivamente, utilizando el protocolo de AGRRA (Cuadro 8).
CUADRO 7

Densidad (individuos $/ 10 \mathrm{~m}^{2}$ ) de erizos en Meager Shoal

TABLE 7

Sea urchin density (ind/10 $\mathrm{m}^{2}$ ) in Meager Shoal

$\begin{array}{lcc}\text { Diadema antillarum } & 4.8 \pm 3.8 & 5.3 \pm 5.5 \\ \text { Echinometra viridis } & 20.0 \pm 18.5 & 0.7 \pm 0.8 \\ \text { Eucidaris tribuloides } & 0.7 \pm 0.8 & 0.2 \pm 0.6\end{array}$


CUADRO 8

Densidad y tamaño medio de los peces arrecifales de Meager Shoal, 2004 (protocolo AGRRA)

TABLE 8

Density and mean size for reef fish in Meager Shoal, 2004 (AGRRA protocol)

$\begin{array}{lcc} & \begin{array}{c}\text { Acanthurus } \\ \text { coeruleus } \\ \text { (cirujano) }\end{array} & \begin{array}{c}\text { Cephalopholis } \\ \text { cruentata } \\ \text { (mero) }\end{array} \\ \text { Densidad }\left(\# / 100 \mathrm{~m}^{2}\right) & 0.33 & 1.67 \\ \text { Tamaño }(\mathrm{cm}) & 15.50 & 12.17\end{array}$

CUADRO 9

Abundancia relativa de peces arrecifales en Meager Shoal, 2004 (protocolo REEF)

TABLE 9

Relative abundance of reef fish in Meager Shoal, 2004 (REEF protocol)

\begin{tabular}{llc}
\multicolumn{1}{c}{ Especies } & \multicolumn{1}{c}{ Familia } & $\begin{array}{c}\text { Abundancia } \\
\text { relativa }\end{array}$ \\
Acanthurus coeruleus & Acanthuridae & $\mathrm{P}$ \\
Chaetodon capistratus & Chaetodontidae & $\mathrm{P}$ \\
Amblycirrhitus pinos & Cirrhithidae & $\mathrm{P}$ \\
Megalops atlanticus & Elopidae & $\mathrm{S}$ \\
Holocentrus rufus & Holocentridae & $\mathrm{S}$ \\
Bodianus rufus & Labridae & $\mathrm{P}$ \\
Thalassoma bifasciatum & Labridae & $\mathrm{P}$ \\
Ocyurus chrysurus & Lutjanidae & $\mathrm{P}$ \\
Abudefduf saxatilis & Pomacentridae & $\mathrm{P}$ \\
Stegastes partitus & Pomacentridae & $\mathrm{P}$ \\
Stegastes fuscus & Pomacentridae & $\mathrm{M}$ \\
Odontoscion dentex & Sciaenidae & $\mathrm{P}$ \\
Cephalopholis cruentata & Serranidae & $\mathrm{P}$ \\
Canthigaster rostrata & Tetraodontidae & $\mathrm{P}$
\end{tabular}

( $\mathrm{S}=$ Solo un individuo; $\mathrm{P}=$ Pocos: $2-10$ individuos; $\mathrm{M}=$ Muchos: 11-100 individuos; $\mathrm{A}=\mathrm{Abundantes:}>100$ individuos).

Mientras que usando el protocolo de REEF los pomacéntridos resultaron ser los peces dominantes en Meager Shoal (Cuadro 9).

Biomasa de algas: La biomasa de algas fue de $1.92 \pm 1.29 \mathrm{~g} / 100 \mathrm{~m}^{2}$ en 2000 . Las algas tapete $(<1 \mathrm{~cm})$ eran las más dominantes y había muy pocas macroalgas.

Materia particulada en suspensión (MPS): La MPS en 1999 era de $18.7 \pm 3.5 \mathrm{mg} / \mathrm{l}$ y $19.7 \pm 8.8 \mathrm{mg} / 1$ en 2003 .

\section{DISCUSIÓN}

La temperatura del agua en Cahuita está dentro del ámbito aceptable para el crecimiento del coral, sin embargo los corales están viviendo en su nivel superior de tolerancia y en el 2003 hubo un evento de calentamiento que pudo haber ocasionado blanqueamiento. La materia particulada en suspensión es la principal fuente de estrés y en 10 años aumentó de $9 \mathrm{mg} / \mathrm{l}$ hasta $20 \mathrm{mg} / \mathrm{l}$ (Cortés 1994). Aunque la proporción de colonias enfermas disminuyó en un $14 \%$, la cobertura de coral vivo y de algas coralinas es muy baja, y la cobertura de algas tapete no coralinas dominan el sustrato y aumentó de 2000 a 2004. Desde la pérdida de la cobertura de coral vivo a finales de 1970 de 40 a $11 \%$ (Cortés 1994) el arrecife muestra sólo una leve recuperación (17\%) posiblemente relacionada con el aumento del erizo Diadema que mantiene baja la cobertura por macroalgas (Edmunds y Carpenter 2001). La recuperación de la cobertura de coral por lo general es lenta y por otro lado la baja cobertura de algas coralinas podría estar afectando el reclutamiento de algunos corales (Morse et al. 1988).

El erizo D. antillarum, una especie herbívora clave, era común en Cahuita (43 ind/ 10m²; Cortés 1981) antes de la mortalidad masiva de 1983 en la región Caribe (Lessios et al. 1984, Murillo y Cortés 1984). La densidad de Diadema disminuyó con dicho evento (0.01 ind $/ 10 \mathrm{~m}^{2}$; Cortés 1994) pero al parecer se está recuperando, ya que las densidades son mayores que hace 10 años, esto es, $0.1-1$ ind/10 $\mathrm{m}^{2}$ en los sitios más someros de Cahuita (Fonseca 2003) y 5-6 ind $/ 10 \mathrm{~m}^{2}$ en Meager Shoal, de 2000 a 2004. Además, se observó la liberación de gametos de varios individuos en 2000 (Fonseca 2003). En los últimos cinco años las 
densidades de $E$. viridis disminuyeron significativamente por razones desconocidas.

La densidad de peces es baja posiblemente por el bajo relieve de este arrecife (Phillips y Pérez-Cruet 1984), y disminuyó de $20 \mathrm{ind} / 100 \mathrm{~m}^{2}$ en 1999 a 2 ind $/ 100 \mathrm{~m}^{2}$ en 2004. Estos son promedios de densidades tomando en cuenta sólo las especies clave, usando la metodología AGRRA, por lo que se está subestimando la contribución de otras especies a la densidad total; sin embargo se asume que el patrón se mantiene. La diversidad y densidad de peces resultó baja en Cahuita (Fonseca y Gamboa 2003), en comparación con otros sitios del Caribe (49 ind $/ 100 \mathrm{~m}^{2}$; Ginsburg y Lang 2003), posiblemente relacionado con la condición tan pobre de los arrecifes de Costa Rica. La presión por pesca es relativamente baja ya que sólo la pesca con línea fue permitida después de la creación del parque (Fonseca y Gamboa 2003) y a partir del año 2005 la pesca se prohibió completamente dentro de áreas protegidas.

La cobertura de coral vivo encontrada en Meager Shoal en 1999 resultó más baja que la informada ahora (Fonseca 2003), pero esto se debe a que en esa ocasión se utilizó la metodología AGRRA, que consiste de transectos en línea recta que no siguen el contorno arrecifal, por lo que la cobertura se subestima en comparación con la metodología de CARICOMP y los resultados no son comparables. Sin embargo, Fonseca (2003) mostró que la cobertura de coral vivo era relativamente más baja en Meager Shoal que en sitios más someros probablemente por la cantidad reducida de luz, relacionada con una mayor profundidad y MPS. También se interpretó que en Meager Shoal la presencia de Diadema en mayores densidades puede ser responsable por la baja cobertura por macroalgas y el leve aumento en la cobertura de coral vivo (Edmunds y Carpenter 2001, Fonseca 2003), a pesar de que la cobertura por algas tapete aumentó (Cuadro 3).

Comparando nuestros datos del año 2000 con datos publicados para otros sitios de CARICOMP en ese mismo año (base de datos
CARICOMP 2000), la cobertura de coral vivo era baja (14.5\%) pero no la más baja, ya que en el resto del Caribe presentó un ámbito entre $1.1 \%$ en Puerto Morelos, México, y $44.8 \%$ en La Parguera, Puerto Rico; lo encontrado en Costa Rica resultó similar a lo encontrado en Carrie Bow Cay, Belize, y Discovery Bay, Jamaica (ambos 12.1\%). La cobertura de coral vivo fue baja $(<50 \%)$ en todos los sitios de CARICOMP desde los años 80. Por otro lado, la cobertura por algas resultó intermedia en Costa Rica (62.9\%), en comparación con el rango de CARICOMP que varió de $0.1 \%$ en Cayo Sombrero, Venezuela, y $92.8 \%$ en Puerto Morelos, México, y también similar a Carrie Bow Cay (53.7 \%) y Discovery Bay (56.1\%). La densidad de D. antillarum (5 ind $/ 10 \mathrm{~m}^{2}$ ) fue baja, en comparación con la densidad máxima encontrada en Barbados (19.4 ind $/ 10 \mathrm{~m}^{2}$ ) pero similar a lo encontrado en otros sitios como Jamaica. Las poblaciones de E. viridis también son bajas. La biomasa de algas es baja ya que en Meager Shoal las dominantes son las algas tapete de menos de $1 \mathrm{~cm}$ de alto, y esto se debe probablemente a que la población de erizos Diadema las mantienen bajo control (Fonseca 2003).

En conclusión el estado de los arrecifes coralinos de Cahuita es similar al de Carrie Bow Cay y Discovery Bay, y al igual que otros sitios de CARICOMP no muestra una recuperación significativa en cuanto a la cobertura de coral vivo desde los años 80 . Esto quiere decir que el grado de resiliencia o recuperación de estos arrecifes es baja (Nyström et al. 2000, Nyström y Folk 2001). El principal problema de éste y otros arrecifes continentales o alrededor de islas grandes y sobrepobladas es la sedimentación y contaminación crónicas acarreadas por escorrentía y corrientes desde suelos deforestados y sobre-explotados en las costas y los bosques riverinos fuera de áreas protegidas (Richmond 1993). Por otro lado la actividad turística está aumentando y no se tienen suficientes regulaciones. Recomendamos reforzar el manejo del Parque Nacional Cahuita, como por ejemplo la implementación de boyas de anclaje para 
turismo y pesca, y tomar medidas de regulación de las actividades de desarrollo fuera del área protegida, especialmente a lo largo de los ríos y costas corriente arriba de este arrecife.

\section{AGRADECIMIENTOS}

Este proyecto fue financiado por CARICOMP, CONICIT, UNEP, Programa cooperativo de desarrollo de la investigación US-ISRAEL y la Vicerrectoría de Investigación, Universidad de Costa Rica. Además, se agradece profundamente a José Saballo y el resto del personal del Parque Nacional Cahuita, Justo López y la familia Mairena por su apoyo.

\section{RESUMEN}

Los arrecifes coralinos del Parque Nacional Cahuita, en la costa Caribe de Costa Rica, específicamente en el sitio CARICOMP llamado Meager Shoal, han sido monitoreados desde 1999. Bases de datos completas de los años 2000 y 2004, muestran que la cobertura de coral viva se mantuvo baja con un aumento menor al $3 \%$ (15-17\%), pero la cobertura de algas no coralinas aumentó mucho (63-74\%) y la cobertura de algas coralinas disminuyó significativamente $(17-5 \%) \quad(\mathrm{p}<.05)$. La proporción de colonias coralinas afectadas por enfermedades, blanqueamiento y otros daños disminuyó de $24 \%$ en el 2000 a $10 \%$ en el 2004, pero la diferencia no resultó significativa. Las densidades de Diadema antillarum aumentaron mientras que las de Echinometra viridis disminuyeron significativamente. El arrecife coralino del Parque Nacional Cahuita continúa siendo afectado por sedimentos terrígenos y no presenta una recuperación significativa desde finales de los años 70 .

Palabras clave: arrecife de coral, monitoreo, CARICOMP, Cahuita, Caribe, Costa Rica.

\section{REFERENCIAS}

CARICOMP. 1997. Caribbean coastal marine productivity (CARICOMP): a research and monitoring network of marine laboratories, parks, and reserves. Proc. $8^{\text {th }}$ Int. Coral Reef Symp., Panamá, 1: 641-646.

CARICOMP. 2002. Status and temporal trends at CARICOMP coral reef sites. Proc. $9^{\text {th }}$ Int. Coral Reef Symp., Bali, Indonesia 1: 325-330.
Connell, J.H. 1978. Diversity in tropical rain forests and coral reefs. Science 199: 1302-10.

Cortés, J. 1992. Organismos de los arrecifes coralinos de Costa Rica: V. Descripción y distribución geográfica de hidrocorales (Cnidaria; Hydrozoa: Milleporina \& Stylasterina) de la costa Caribe. Brenesia 38: 45-50.

Cortés, J. 1994. A reef under siltation stress: a decade of degradation, p. 240-246. In R.N. Ginsburg (compiler). Proc. Colloquium on Global Aspects of Coral Reefs: Health, Hazards and History, 1993. RSMAS, Univ. Miami, Miami, Florida, EEUU.

Cortés, J. 1998. Cahuita and Laguna Gandoca, Costa Rica, p. 107-113. In B. Kjerfve (ed.). CARICOMP. Caribbean Coral Reef, Seagrass and Mangrove Sites. UNESCO, Paris, Francia.

Cortés, J. \& H. Guzmán. 1985. Organismos de los arrecifes coralinos de Costa Rica: III. Descripción y distribución geográfica de corales escleractinios (Cnidaria: Anthozoa: Scleractinia) de la costa Caribe. Brenesia 24: 63-124.

Cortés, J. \& C.E. Jiménez. 2003. Past, present and future of the coral reefs of the Caribbean coast of Costa Rica, p. 223-239. In J. Cortés (ed.). Latin American Coral Reefs. Elsevier Science B.V., Amsterdam, Holanda.

Cortés, J. \& M.J. Risk. 1985. A reef under siltation stress: Cahuita, Costa Rica. Bull. Mar. Sci. 36: 339-356.

Edmunds, P.J. \& R.C. Carpenter. 2001. Recovery of Diadema antillarum reduces macroalgal cover and increases abundance of juvenile corals on a Caribbean reef. Proc. Natl. Acad. Sci. USA 98: 5067-5071.

Fonseca, A.C. 2003. A rapid assessment at Cahuita National Park, Costa Rica, 1999 (Part I: stony corals and algae), p. 249-257. In J.C. Lang (ed.).Status of Coral Reefs in the Western Atlantic: Results of Initial Surveys, Atlantic and Gulf Rapid Reef Assessment (AGRRA) Program. Atoll Res. Bull. 496.

Fonseca, A.C. \& J. Cortés. 2002. Land use in the La Estrella River basin and soil erosion effects on the Cahuita Reef system, Costa Rica, p. 68-82. In B. Kjerfve (eds.). Land-Ocean Interactions in the Coastal Zone (LOICZ). Core project of the International Geosphere Biosphere Programme: a study of Global Change (IGBP). Caribbean Basins (CARIBAS). LOICZ Rep. Stud., 27.

Fonseca, A.C. \& C. Gamboa. 2003. A rapid assessment at Cahuita National Park, Costa Rica, 1999 (Part 2: Reef fishes), p. 258-266. In J.C. Lang (ed.). Status of Coral Reefs in the Western Atlantic: Results of Initial Surveys, Atlantic and Gulf Rapid Reef Assessment (AGRRA) Program. Atoll Res. Bull. 496. 
Ginsburg, R.N. \& J. Lang. 2003. Foreword, p. vii-xiii. In J.C. Lang (ed.). Status of Coral Reefs in the Western Atlantic: Results of Initial Surveys, Atlantic and Gulf Rapid Reef Assessment (AGRRA) Program. Atoll Res. Bull. 496.

Guzmán, H.M. \& J. Cortés. 1985. Organismos de los arrecifes coralinos de Costa Rica: IV. Descripción y distribución geográfica de octocoralarios de la costa Caribe. Brenesia 24: 125-174.

Hughes, T.P., A.H. Baird, D.R. Bellwood, M. Card, S.R. Connolly, C. Folke, R. Gosberg, O. Hoehg-Guldberg, J.B.C. Jackson, J. Kleypas, J.M. Lough, P. Marshall, M. Nyström, S.R. Palumbi, J.M. Pandolfi, B. Rosen \& J. Roghgarden. 2003. Climate change, human impacts, and the resilience of coral reefs. Science 301: 929-933.

Hughes, T.P., D.R. Bellwood, C. Folke, R. Steneck \& J. Wilson. 2005. New paradigms for supporting the resilience of marine ecosystems. Trends Ecol. Evol. 20: $380-386$.

Lessios, H.A., D.R. Robertson \& J.D. Cubit. 1984. Spread of Diadema mass mortality through the Caribbean. Science 226: 335-337.

Morse, D.E., N. Hooker, A.N.C. Morse \& R.A. Jensen. 1988. Control of larval metamorphosis and recruitment in sympatric agariciid corals. J. Exp. Mar. Biol. Ecol. 116: 193-217.

Murillo, M.M. \& J. Cortés. 1984. Alta mortalidad en la población del erizo de mar Diadema antillarum
Philippi (Echinodermata: Echinoidea), en el Parque Nacional Cahuita, Limón, Costa Rica. Rev. Biol. Trop. 32: 167-169.

Nyström, M., C. Folke \& F. Moberg. 2000. Coral reef disturbance and resilience in a human-dominated environment. Trends Ecol. Evol. 15: 413-417.

Nyström, M. \& C. Folke. 2001. Spatial resilience of coral reefs. Ecosystems 4: 406-417.

Pandolfi, J.M., R.H. Bradbury, E. Salas, T.P. Hughes, K.A. Bjorndal, R.G. Cooke, D. McArdle, L. McClenachan, M.J.H. Newman, G. Paredes, R.R. Warner \& J.B.C. Jackson. 2003. Global trajectories of the long-term decline of coral reef ecosystems. Science 301: 955-958.

Phillips, P.C. \& M.J. Pérez-Cruet. 1984. A comparative survey of reef fishes in Caribbean and Pacific of Costa Rica. Rev. Biol. Trop. 32: 95-102.

Richmond, R.H. 1993. Coral reefs: present problems and future concerns resulting from anthropogenic disturbance. Amer. Zool. 33: 524-536.

Risk, M.J., M.M. Murillo \& J. Cortés. 1980. Observaciones biológicas preliminares sobre el arrecife coralino en el Parque Nacional Cahuita, Costa Rica. Rev. Biol. Trop. 28: 361-382.

Underwood, A. 1997. Experiments in Ecology: Their Logical Design and Interpretation using Analysis of Variance. Univ. Cambridge, Cambridge, United Kingdom. 504 p. 
